\title{
A Study on Reconstructing Temperature Field
}

\author{
Xian Zhou ${ }^{1, a}$, Wei-gen Wu ${ }^{2, b}$, Gao-yuan Niu ${ }^{1, c}$ and Qiang Wang ${ }^{1, d}$ \\ ${ }^{1}$ School of Electrical and Information Engineering, Xihua University,chengdu 610039,China \\ ${ }^{2}$ School of Electrical and Information Engineering, Panzhihua University,Panzhihua 617000, China

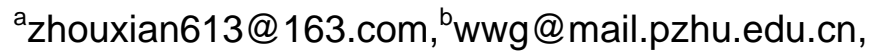 \\ czhang963niu@163.com, ${ }^{\mathrm{c}}$ wangqiang198612@163.com
}

Keywords: three-dimension temperature field,acoustic thermometry,DCT

\begin{abstract}
This paper puts forward a new algorithm based on RBF neural network to reconstruct three-dimensional temperature field.The algorithm uses three-dimension Discrete Cosine Transform on temperature field,and establishes a mapping relation between low order term coefficient vector and sound wave path average temperature vector,then implements the mapping relation using radical basis function neural network.Finally three-dimensional temperature field was reconstructed by using Inverse three-dimension Discrete Cosine Transform.Simulation results show that the algorithm features high precision.
\end{abstract}

\section{Introduction}

Complex temperature field exists in a variety of equipments used for combustion and heating,such as boilers in the power industry and furnaces in metallurgical industry.Reconstructing complex temperature field can realize visualization of measured temperature field and achieve the purposes of detecting and controlling temperature state.Since acoustic thermometry has the advantages of non-contact,real time measure and easily maintained,it provide an effective method for temperature field reconstruction. A fast and accurate algorithm is the key to reconstruct temperature field.On the basis of the previous research,this paper puts forward a new algorithm based on RBF neural network to reconstruct temperature field.

\section{Principle of acoustic thermometry} as $^{[1]}$ :

Speed of sound wave in ideal gas $C$ is a function of gas temperature $T$.It can be expressed

$$
C=\left(\frac{k \bullet R}{M} \bullet T\right)^{\frac{1}{2}}=Z \sqrt{T}
$$

$C$ is the speed of sound wave in ideal gas and its unit is $\mathrm{m} / \mathrm{s} ; R$ is the gas constant with value of $8314.3 \mathrm{~J} / \mathrm{kmolK} ; T$ is the gas thermodynamic temperature; $k$ is the adiabatic index of ideal gases, with value of specific heat at constant pressure to specific heat of constant volume; $M$ is the gas average thousand molecular weight and its unit is $\mathrm{kg} / \mathrm{kmol} ; Z$ is the constants associated with gas.

Sound wave transmitting and receiving devices are installed around the measured area.Average temperature on each path is a function of sound wave travel-time ${ }^{[2]}$ :

$$
T_{m}=\frac{D^{2}}{B \tau^{2}} \cdot 10^{6}-273.16
$$

(2) $T_{m}$ is the average temperature on measured paths; $D$ is the distance of sound wave devices; $B$ is the acoustic constant; $\tau$ is the sound wave travel-time.

By the analysis above, it is concluded that average temperature on measured paths $T_{m}$ can be calculated if both $D$ and $\tau$ are known. 


\section{Acoustic thermometry reconstruction algorithm}

As is shown in Fig 1,36 sound wave transmitting and receiving devices are installed around the measured area(represented by dark spots),dividing this area into 64 parts.After eliminating the sound wave paths on the same side that can not reflect temperature information,172 valid sound wave travel paths are remained.

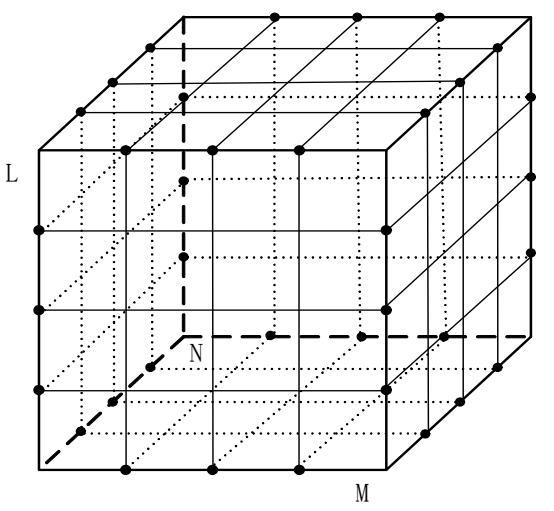

Fig. 1 The location of sound wave transmitting and receiving devices

Supposing temperature in each part is evenly distributed.Reconstructing temperature field means that temperature in each part must be solved.Average temperature on $k$ th path can be expressed as:

$$
T_{m k}=\frac{1}{L_{k}} \sum_{i=1}^{I} \Delta S_{k i} T_{i}, k=1,2,3 \cdots n
$$

$T_{m k}$ is the average temperature on the $k$ th path; $L_{k}$ is the total length of the $k$ th path; $\Delta S_{k i}$ is the length of the $k$ th path in the $i$ th part; $T_{i}$ is the temperature of the $i$ th area; $n$ is the total of the path; $I$ is the number of the parts into which the whole temperature field is divided.

In order to reconstruct temperature field $T(x, y, z)$, three-dimension Discrete Cosine Transform(DCT) is used to transform $T(x, y, z)$ into a representation of coefficient $A$ and basis function. The coefficient matrix of $T(x, y, z) \quad(0 \leq x \leq M-1 ; 0 \leq y \leq N-1 ; 0 \leq z \leq L-1)$ is $A(u, v, w)$, which can be expressed as:

$$
\begin{aligned}
& A(u, v, w)=\sqrt{\frac{2^{3}}{L M N}} c(u) c(v) c(w) \times \sum_{x=0}^{M} \sum_{y=0}^{N} \sum_{z=0}^{L-1} T(x, y, z) \cos \left[\frac{(2 x+1) \pi u}{2 M}\right] \\
& \times \cos \left[\frac{(2 y+1) \pi v}{2 N}\right] \times \cos \left[\frac{(2 z+1) \pi w}{2 L}\right] \\
& u=0,1, \cdots M-1 ; \quad v=0,1, \cdots N-1 ; \quad w=0,1, \cdots L-1 \\
& c(u)=\left\{\begin{array}{lc}
\frac{1}{\sqrt{2}} & u=0 \\
1 & \text { others }
\end{array} \quad c(v)=\left\{\begin{array}{ll}
\frac{1}{\sqrt{2}} & v=0 \\
1 & \text { others }
\end{array} \quad c(w)= \begin{cases}\frac{1}{\sqrt{2}} & w=0 \\
1 & \text { others }\end{cases} \right.\right.
\end{aligned}
$$

Considering actual temperature distribution,we abandon high order term coefficients and reserve low order term coefficients where energy is centralized.Low order term coefficients will be good approximations to reflect original temperature field $T(x, y, z)$.Then original temperature field is reconstructed by Inverse Three-dimension Discrete Cosine Transform, which can be expressed as:

$$
\begin{aligned}
& T(x, y, z)=\sqrt{\frac{2^{3}}{L M N}} c(u) c(v) c(w) \times \sum_{x=0}^{M-1} \sum_{y=0}^{N-1} \sum_{z=0}^{L-1} A(u, v, w) \times \cos \left[\frac{(2 x+1) \pi u}{2 M}\right] \\
& \times \cos \left[\frac{(2 y+1) \pi v}{2 N}\right] \cos \left[\frac{(2 z+1) \pi w}{2 L}\right]
\end{aligned}
$$


Inputs $\bar{t}$ consists of average temperature $\overline{t_{1}}, \overline{t_{2}}, \ldots \overline{t_{n}}$. Low order term coefficients vectors $A$ is outputs. $A$ and $\bar{t}$ mapping relationship can be expressed as:

$$
A=f(\bar{t})
$$

Due to the fact that training function of RBF neural network has the advantages of fast convergent rate and good approximation ability,it can be used to fulfill the mapping relationship of Eq. 6.The RBF neural network takes $\bar{t}$ as inputs and takes $A$ as outputs.After $\bar{t}$ is obtained, $A$ can be solved by using RBF Neural Network.Inverse three-dimension Discrete Cosine Transform is used to reconstruct original temperature at last.

\section{Structure of RBF neural network and its learning algorithm}

As is shown in Fig 2,RBF neural network is composed of one input layer,one hidden layer and one linear output layer.The activation function of hidden layer nodes is $\phi_{j}(x)$,having the property of radial symmetry.Output $y$ is a linear combination of the activation function output.That is:

$$
y(x)=\sum_{j=0}^{c} w_{j} \phi_{j}(x) \text {, Where } \phi_{j}(x)=\exp \left(\frac{\left\|x-v_{j}\right\|}{2 \delta_{j}^{2}}\right)
$$

$w_{j}$ is the weight between the $j$ th hidden layer nodes and output layer nodes. $v_{j}$ is the center of the $j$ th hidden layer nodes(also known as center vectors ), and $\delta_{j}$ is the width of the $j$ th hidden layer nodes.

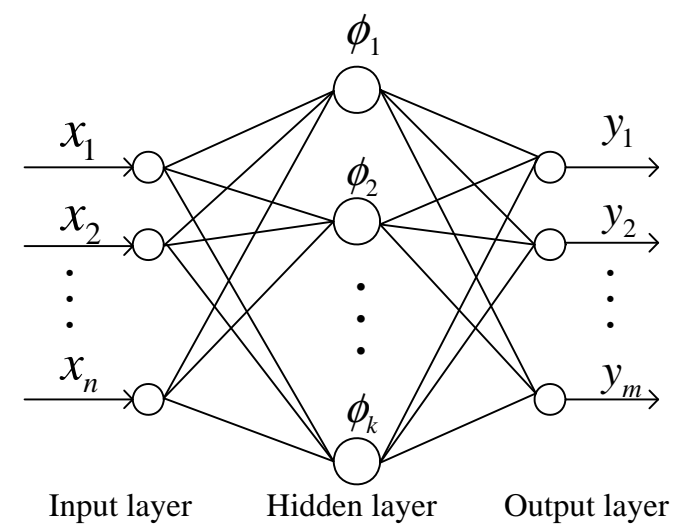

Fig. 2 The structure of RBF neural network

There are two phases of RBF network learning algorithm:

1) calculate the parameters to kernel function including $v_{j}$ and $\delta_{j}$.The solution usually adopted is K-means clustering algorithm.

2) get the weights matrix $W$.The solution usually adopted is least square method.In the two parameters $v$ and $\delta$,calculating $\delta$ are relatively complex,but changes in $\delta$ have little impact on RBF neural network.So we can roughly assign a value based on the sample data,provided that the matrix which consists of hidden layer outputs is not ill - conditioned.

The number of centers $v$ must be determined before we use k-means algorithm to get centers $v$.After initializing centers $v$, we groups all of the $x_{i}$ and $v_{j}$ to make $\left\|x_{i}-v_{j}\right\|=\min _{j}\left\|x_{i}-v_{j}\right\|$. For each group of $v_{j}$,we continue to calculate its mean value which is used as the new center until each center does not change.

Once centers $v$ are calculated,RBF network hidden layer output is determined.The values of all elements in the matrix can be calculated using least square method because hidden layer is linear with output layer,and they are only a difference of weight matrix product factor. 


\section{The simulation results and corresponding analysis}

A simulated temperature field is constructed in a $10 m \times 10 m \times 10 m$ space as:

$$
T(x, y, z)=1200+300 \sin (0.5 x) \sin (0.6 y) \sin (0.7 z)
$$

$M, N$ and $L$ are equal to 10 in reconstructing simulate temperature field - that is,simulate temperature field is divided into 1000 parts.Three-dimension Discrete Cosine Transform coefficients is $M \times N \times L$ matrices.

Fig 3 shows the corresponding coefficients $A(u, v, w)(u, v$ and $w$ belong to 0 to 9$)$.As can be seen from Fig. 3,the energy of coefficients concentrates in just $0 \leq u \leq 3,0 \leq v \leq 3,0 \leq w \leq 3-$ that is,the 64 coefficients form $A(0,0,0)$ to $A(3,3,3)$ can reflect original temperature field $T(x, y, z)$ quite accurately.So we take the number of DCT coefficients as 64 - that is,the number of neural network output is 64 .

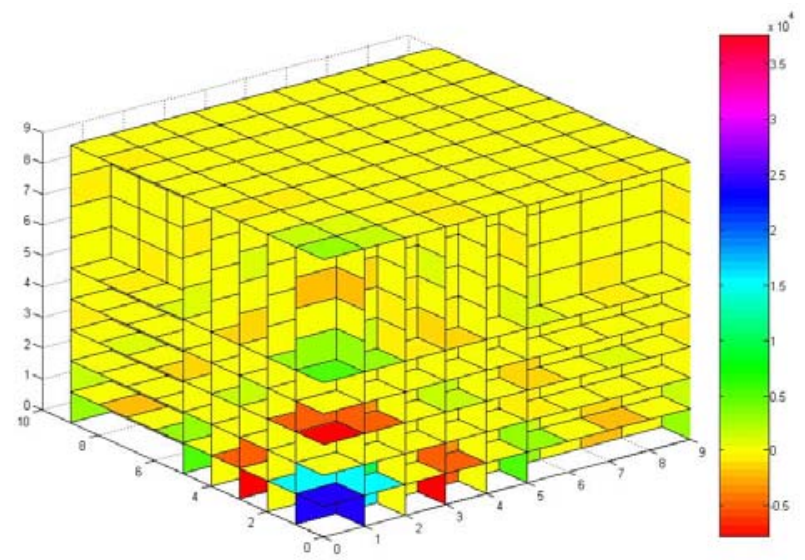

Fig. 3 Three-dimension Discrete Cosine Transform coefficients

Fig. 4 shows simulated temperature field,and Fig. 5 shows reconstructed temperature field using new algorithm $\left({ }^{\circ} \mathrm{C}\right)$. The results were evaluated by using mean error and root mean square error.Root mean square error is defined as ${ }^{[3]}$ :

$$
R M S E=\frac{1}{\bar{T}} \sqrt{\frac{1}{M N L} \sum_{i, j, k=1}^{M, N, L}[\hat{T}(i, j, k)-T(i, j, k)]^{2}} \times 100 \%
$$

$\bar{T}$ is the average temperature of simulated temperature field. $\hat{T}(i, j, k)$ is the average temperature of reconstructed temperature field.

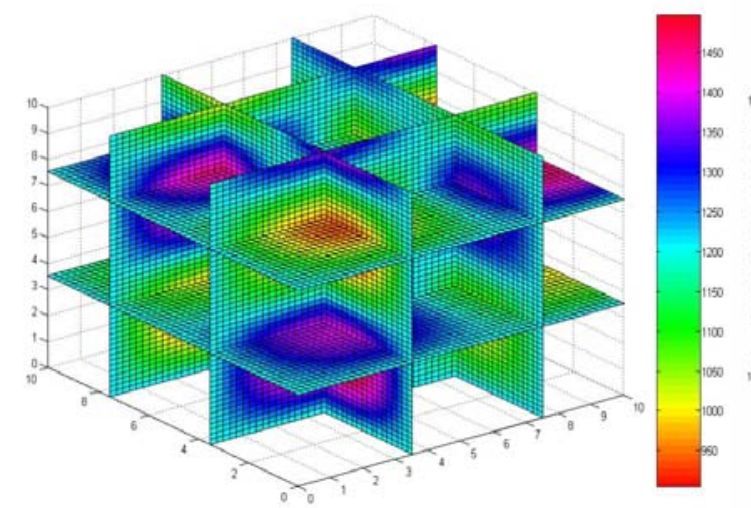

Fig. 4 Simulated temperature field

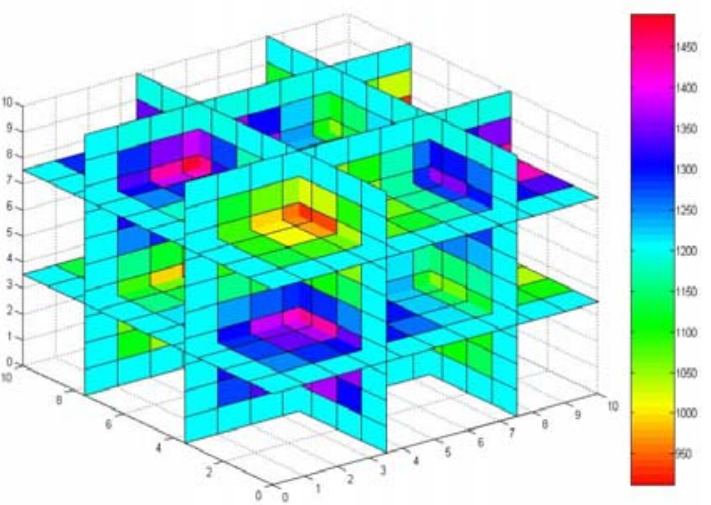

Fig. 5 Reconstructed temperature field

Table 1 The reconstruction results

\begin{tabular}{cccccc}
\hline & $\begin{array}{c}\text { mean } \\
\text { temperature }\end{array}$ & $\begin{array}{c}\text { highest } \\
\text { temperature }\end{array}$ & $\begin{array}{c}\text { lowest } \\
\text { temperature }\end{array}$ & $\begin{array}{c}\text { mean } \\
\text { error }\end{array}$ & $\begin{array}{c}\text { mean square } \\
\text { root }\end{array}$ \\
\hline error \\
\hline simulated & $1101.2^{\circ} \mathrm{C}$ & $1500.0^{\circ} \mathrm{C}$ & $900.0^{\circ} \mathrm{C}$ & - & - \\
reconstructed & $1048.5^{\circ} \mathrm{C}$ & $1426.2^{\circ} \mathrm{C}$ & $871.7^{\circ} \mathrm{C}$ & 4.8 & 4.2 \\
\hline
\end{tabular}


The reconstruction results are shown in Table 1.It's concluded that the proposed algorithm is simple and effective.

\section{Conclusion}

At present,research on temperature field reconstruction is little and mainly targets two-dimensional temperature field reconstruction ${ }^{[4-5]}$. The paper researches into three-dimensional temperature field reconstruction.Just as simulation analysis shows,compared with two-dimensional temperature field reconstruction, three-dimensional temperature field reconstruction can obtain much more information, so it has higher precision relatively and more powerful anti-interference capacity.

\section{References}

[1] Helmut S.Waveform inversion in acoustic pyrometry[A].The $1^{\text {th }}$ world Congress on Industrial Process Tomography[C].Buxton Greater Manchester,April,1999:14-17

[2] Tian F.Temperature field reconstruction algorithm based on RBF neural network[J].Chinese Journal of Scientific Instrument.2006,27(11):1460-1464.

[3] MAUJRO B,EMANUELE A.An acoustic pyrometer system for tomographic thermal imaging in power plant boilers[J].IEEE Transactions on Instrumentation and Measurement, 1996,45(1):159-161.

[4] Shogo T.Measurement of temperature distribution in boilers using acoustic sensors[A].SICE[C].1999,35(9):1147-1153.

[5] Fumio I,Masayasu S.Fundamental studies of acoustic measurement and reconstruction combustion temperature in lager boilers[J].Trans,Japan Soc.Mech.Eng,1985,B53:1610-1614. 